\title{
Research and Application of the Multiple Meter Data Acquire Technical
}

\author{
Du Yan ${ }^{1+}$, Wang Zhe Long ${ }^{1}$, Guo Liang ${ }^{2}$ \\ ${ }^{1}$ State Grid ShanDong Electric Power Research Institute \\ ${ }^{2}$ State Grid ShanDong Electric Power Company
}

\begin{abstract}
This paper analyzes the current situation of the development of watt-hour meter,water meter, gas meter, heat meter. Introduced the framework of electric energy data acquire system. Proposed two kinds of reform plan of multiple meter data acquire construction, one plan is increase communication interface converter on the original architecture which can collection water meter, gas meter and heat meter data and then transmit to the concentrator, another plan is using the micro power wireless collector or micro power wireless watt-hour meter as relay to collect water meter, gas meter and heat meter data and then transmit to the concentrator. Finally, according to the field application of the pilot reform project to summary the achievements and problems,, and given the development trend of the multiple meter data acquire.
\end{abstract}

Keywords: Multiple meter data acquire, Communication protocol, Communication interface converter, M-BUS.

\section{Introduction}

In order to promote the development of "smart city", comprehensive utilization of public resources, solve the problem of "last mile" for the people service, Relying on the intelligent watt-hour meter and electric energy data acquisition system covering a wide range of data acquire terminal and communication resources, and building electricity, water, gas, heat multiple meter data collection application system, to promote the development of smart electricity and two-way interaction. At present, the State Grid Corporation has priority in some residential district which have the conditions to carry out the four meter data acquire demonstration project construction, in accordance with the "first demonstration, from easy to difficult; resource sharing, the lowest cost; unified standards, typical scheme; multi-point layout, scale advance; innovation model, achieve win-win" principle, plans to build more than one hundred experiment in national.

\section{Communication Technology Used of the Tour Meter}

Communication technology is the important basis to realize four meter data acquire, It determines the operating principle of the system, and also affects the operating efficiency and reliability of the system. The watt-hour mete adopted communication protocol is DL/T645-2007 protocol, the data acquire terminal adopted communication protocol is Q/GDW 376.1 and Q/GDW 1376.1. Water meter mainly through the MBUS and RS-485 communication [1], the thermal energy meter communication mode is M-BUS [2], gas meter mainly adopts micro power wireless communications at present [3], water meter, heat meter and gas meter communication protocol are reference to CJ/T188-2004 and expand the agreement. In summary, the four meter data acquire communication technology are mainly adopts the M-BUS bus, RS-485, micro power wireless and power carrier etc.

\subsection{M-BUS}

\footnotetext{
+ Corresponding author. Tel.: + 15069111652; fax: +0531-67982727.

E-mail address: 461849930@qq.com.
} 
M-BUS is a kind of hierarchical communication system controlled by the host computer, which is composed of a host, a slave computer and two connecting cable. can not directly exchange information between slave computer, and can only be forwarded through the host. M-BUS communication technology data transfer rate of up to 300 9600bps and maximum transmission distance is about 1000 meters, its advantage is:

- The wiring is simple, only two communication lines, the bus has no polarity, no special requirements for wiring way, and can be connected in series or parallel.

- Can realize data acquire terminal remote power supply for measuring equipment through the communication line, suitable for water, gas and heat meter, which is itself no power supply of the meter.

- Communication stability, anti-interference ability is strong, can ensure the success rate of communication.

Its disadvantages are:

- Need site wiring, household wiring may damage the existing home devices.

- After long time operation, it may appear that the connection of the communication line is oxidized, which makes the maintenance of the later stage is complicated.

\subsection{RS-485}

The RS-485 is a kind of communication technology using differential voltage signal line for signal transmission. The data transfer rate is below $1 \mathrm{Mbps}$, the maximum coverage distance is 1200 meters.

Its advantage is:

- High speed of communication, using differential signal for data transmission, anti-interference ability is strong.

- Communication stability is good.

Its disadvantages are:

- Installation requires a lot of wiring, construction is complicated.

- Due to the RS - 485 communication line don't have the power supply ability, therefore it unable direct supply power to water meter, gas meter and heat meter, need to be equipped with an external power supply or backup power supply, led to the cost obviously improve of the equipment.

\subsection{Micro Power Wireless}

Micro power wireless communication technology refers to the transmission power is less than $50 \mathrm{mw}$,covering the range of hundreds meters, use of $470 \mathrm{MHz} 510 \mathrm{MHz}$ frequency band, have ad-hoc network function of wireless communication technology, the communication rate can be up to $10 \mathrm{kbps}$.

Its advantages are:

- Without need to field wiring, field construction is convenient.

- Don't need to pay the communication fee to the telecom operators.

- Flexible network, data transfer rate is higher.

Its disadvantages are:

- The communication effect is poor in the large range of area or electromagnetic shielding.

- Vulnerable to the influence of the weather environment, anti-interference ability is poor.

- It unable direct supply power to water meter, gas meter and heat meter, need to be equipped with external power or backup power supply.

\subsection{Power Carrier}

Power line carrier communication technology refers to the use of power line of power frequency transmission of high frequency weak signal. Power line carrier communication is generally used in $3 \sim 500 \mathrm{MHz}$ (narrowband carrier) or $2 \sim 30 \mathrm{MHz}$ (broadband carrier) power line spectrum resources, data transmission rate up to $1 \mathrm{kbps}$.

Its advantage is: 
- Relying on the power line, without laying telecommunication lines, save a certain cost.

- Electric power area management mode can be introduced.

Its disadvantages are:

- The communication performance is seriously affected by the noise of the grid, and the stability is poor.

- Itself needs to be equipped with external power supply.

\section{Technical Solutions of Multiple Meter Data Acquire}

Based on the original electric energy data acquisition system, and satisfy the diversification of unity of four meter, technical solutions of four meter data acquire should consider reuse the data acquire terminal, remote channel and master station of the electric energy data acquisition system, at the lowest cost and reform realize applications of the four meter data acquire technical, this paper introduces two kinds of solution that increase communication interface converter and upgrade wireless module.

\subsection{Electric Energy Data Acquisition Architecture}

The electric energy data acquisition system is composed of master station layer, remote communication layer, data acquire terminal layer, local communication layer, and the watt-hour meter layer. Master station through wireless public network, $230 \mathrm{MHZ}$ wireless private network, optical fiber private network and other remote communication technology interaction with data acquire terminal; data acquire terminal communication with watt-hour meter through narrowband power line carrier, broadband power line carrier, micro power wireless, RS-485 and other local communication technology. [4]The framework of electric energy data acquire system is shown in Figure 1.

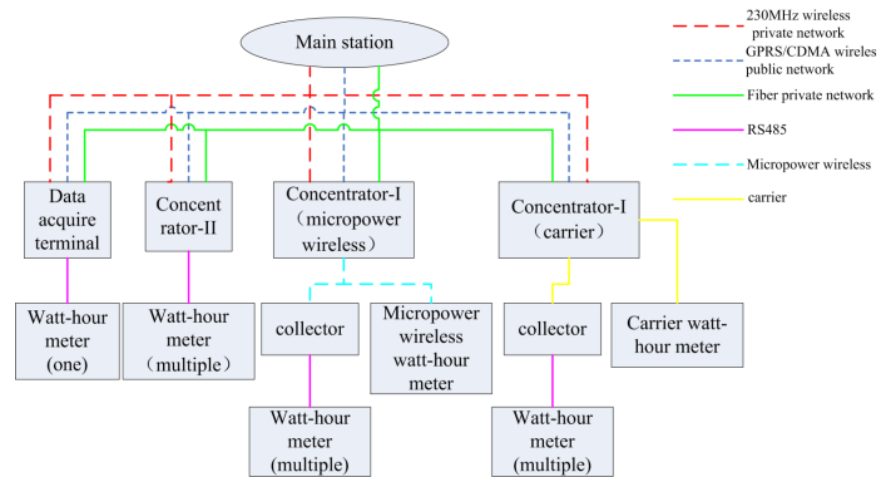

Fig.1: The framework of electric

energy data acquire system.

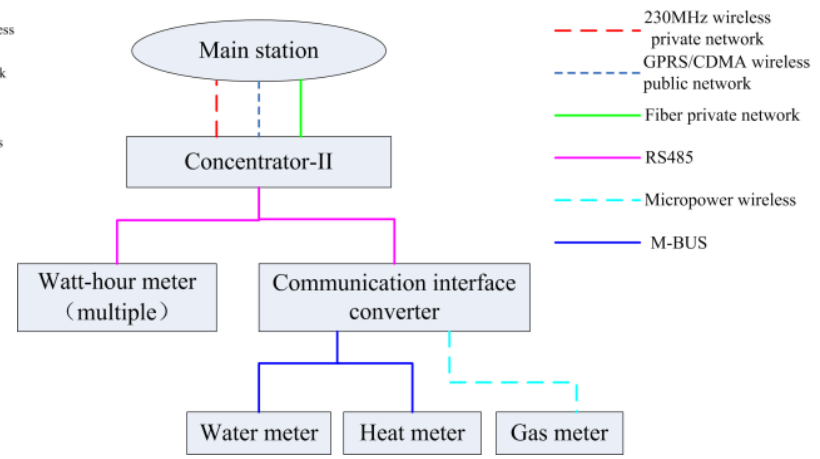

Fig.2: The framework data acquire system with communication interface converter.

The data acquire terminal collect electric data information of the watt-hour meter through the RS485, and reported to the main station by the $230 \mathrm{MHz}$ wireless private network, GPRS wireless public network and fiber private network. The concentrator-II collect electric data information of the watt-hour meter through the RS485, generally a concentrator-II can connect dozens of only to more than one hundred watt-hour meter. GPRS watt-hour meter communication with main station by GPRS wireless public network .For three layer structure of concentrator-I, it communication with collectors which the concentrator-I connect through the micro power wireless or powerline carrier, a concentrator-I can connect multiple collectors, collector collect electric data information of the watt-hour meter through the RS485.For two layer structure of concentrator-I, it communication with Micro power wireless watt-hour meter or Carrier watt-hour meter by the micro power wireless or powerline carrier.

\subsection{Increase Communication Interface Converter}

Due to the difference of the structure of the electric energy data acquire system, so the reform scheme of four meter data acquire is different which based on different structure of the electric energy data acquire system. Acquisition system for concentrator-II, which is based on the existing collection devices, increase the communication interface converter between the concentrator and water meter, gas meter, heat meter, it will transform different communication protocol to the DL/T 645-2007 protocol. [5-6] Through the 
technical proposal, only need to upgrade software of the concentrator-II, keep original position of the watthour meter, water meter, gas meter and heat meter, increase communication interface converter, the cost of reform is low, while achieving hardware isolation of the watt-hour meter and water meter, gas meter, heat meter, ensure that the other meter does not affect the remote acquisition of the watt-hour meter, the structure of after reform is shown in figure 2.

After the reform of the four meter data acquire system need to upgrade the original concentrator-II, on the basis of realize the original power data acquisition, upgrade the protocol parsing layer, increases the data acquisition function and storage function of the water meter, gas meter and heat meter; communication interface converter can converted protocol of the meters which adopt M-BUS and Micro power wireless technology, and realize four meter data acquire.

\subsection{Full Wireless Technology Solutions}

For concentrator-I, if the water meter and heat meter still using the wired communication, the reform scheme still need to install communication interface converter; if the water meter, gas meter and heat meter using the micropower wireless, and the distance between the water, gas, heat meter and the collector-I or micropower wireless watt-hour meter is close, need to upgrade the micropower wireless module, the structure of after reform as shown in Figure 3 and Figure 4 distance.

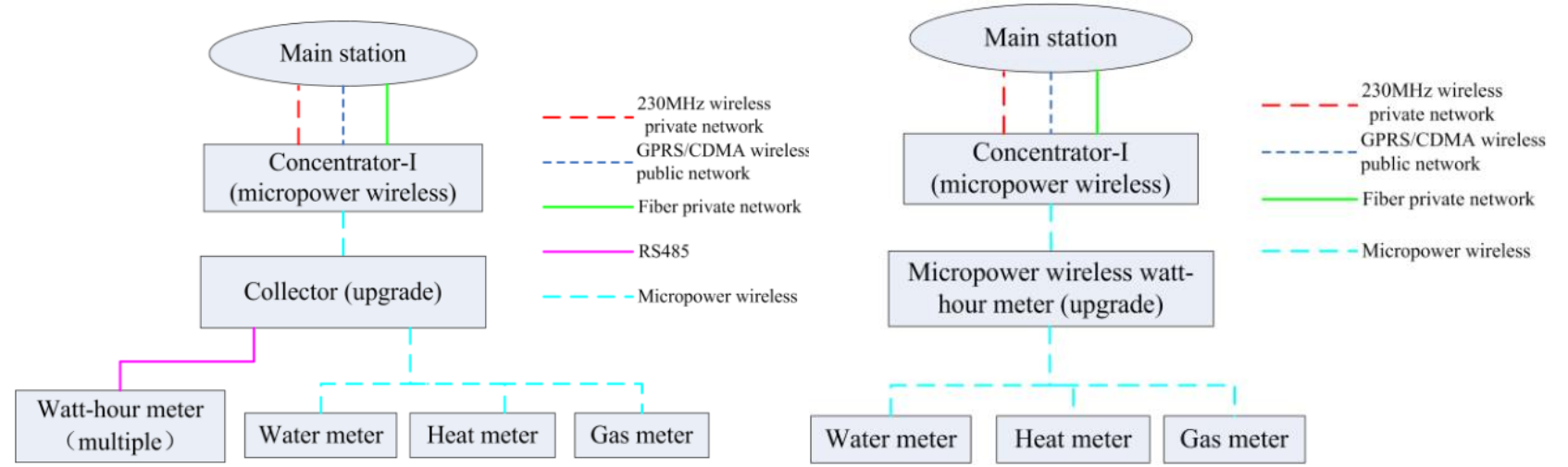

Fig. 3: The framework data acquire system with Collector. Fig. 4: The framework data acquire system without Collector.

Upgrade the software of the collector-I or micropower wireless watt-hour meter In figures 3 and figure 4, realize the data acquire of the water, gas and heat meter, concentrator-I communication with collector-I or micropower wireless watt-hour meter by micropower wireless technical. Due to the micro power wireless water, gas and heat meter are powered by battery and the battery capacity is limited, if the water, gas and heat meter directly with concentrator-I network will consume more energy, and restrict the service life of the water, gas and heat meter, Therefore, the collector-I or the micropower wireless watt-hour meter as the gateway, this scheme can achieve the data forwarding function of water, gas and heat meter, but the water, gas and heat meter can't achieve real-time online communication, only adopt the way of awakening to prolong the service life.

\section{Pilot Effect and Existing Problems}

\subsection{Pilot Effect}

According to the first scheme in this paper which is adds communication interface converter to realized multiple meter data acquire. Communication interface converter has two interface of RS485, two interface of M-BUS, one interface of micro power wireless. It acquire all kinds of data of water meter and heat meter through the M-BUS and acquire all kinds of data of gas meter through micro power wireless, and then convert the CJ188 communication protocol into DL/T645 protocol, communication interface converter transmitted all acquire data to the concentrator by RS485, concentrator transmitted all data to power user electric energy data acquire system through GPRS or fiber. 
Since implemented the reform of the four meter data acquire, the original mechanical meter and local meter upgrade to smart meters and remote automatic reading meter, realize the span of artificial meter reading to automatic meter reading, greatly reducing the workload of artificial meter reading and data entry processing, effectively avoid the errors caused by human factors, improves the accurate, timely and efficiency of meter reading, to promote data automatic collection level of water, gas and heat company. At the same time the construction of the reform for four mete data acquire promote the public utilities data integration reading, realize the customer data sharing, promote the development of the two-way interaction.

\subsection{Existing Problems}

Because the construction of four meter data acquire refer to many industry unit, many types of meter, many technical standards, in promoting the process need to coordination and communication management scheme, technical scheme and economic cost, etc. At present, [7] there are mainly the following problems in the process of reform:

- Four meter management department lack of unified management.

- Pilot products and systems of four meter data acquire do not have universal applicability, only for specific applications.

- Communication architecture complex.

- Hardware, communication mode and communication protocol of measurement meter are uneven.

- The high cost of system operations and maintenance.

\section{Conclusions}

This paper analyzes the current development situation of the watt-hour meter, water meter, gas meter and heat meter, introduced the structure of the electric energy data acquire system, and detail analyzed the two kinds of reform schemes of the four meter data acquire,summarize the pilot effect and existing problems. the next step will be according to the pilot effect, gradually improve the unity on the management and technology, promote development national standards of four meter data acquire technical.

\section{References}

[1] Yao-ling. The technique actuality and development tendency of automatic metering reading for water meter. shanghai measurement and testing.2008, 03: 2-5.

[2] Li-shuang, Yu-yang. Application of remote meter reading system for calorimeter.Gas \& Heat.2009, 29 (05): $20-23$.

[3] Zhang-tao. Application of automatic meter reading to gas charge system. Gas \& Heat. 2010, 30(1): 14-19.

[4] Q/GDW1373-2013.Power user electric energy data acquire system functional specification. Bei-jing:China Electric Power Press,2013.

[5] Ding-yi, Hou-guobing, Qi-sixue, Li-guohua, Zhao-wei. A four-meter collector applied in automatic meter reading. Electrical Measurement \& Instrumentation.2003, 40(455): 11-14.

[6] Cheng Ying-ying, Zhou Feng, Xiao Ji, Jin Zhi-gang. Multi-meter-in One Meter Reading System Based on Neighbor Mesh..Automation \& Instrumentation.2016,(11):51-53

[7] Wu Zhi-ping, Hong Ya-ting, Feng Shu-ning. Research on the new mode of building energy service based on “multi meters combined to one". Power Demand Side Management. 2016, (06):51-53. 